\section{PLTIT: A graphing package for the Apple II series of computers}

\author{
JOHN R. VOKEY \\ University of Lethbridge, Lethbridge, Alberta, Canada
}

PLTIT is a program for plotting data on the Apple II (II +, IIe, IIc, and clones) series of computers. The program is very easy to use; it leads the user through a series of input "screens" that display the current (or default) values of parameters and options and allow the user either to accept the displayed values or to change them to other values; incorrect or nonsensical values are rejected automatically. After moving through the series of screens, the data are plotted and displayed, ready to be saved to disk as a completed plot, to be dumped to a printer, or to serve as the background for some subsequent plot or plots. The user may return to any input screen to experiment with different parameter settings or to transform, scale, or otherwise alter and/or edit the data or finished plot. The program supports four different plot types, and multiple data sets may be plotted simultaneously.

\section{Program Features}

Plot types. PLTIT supports four plot types: polygons, histograms, scatterplots, and pie charts. With the exception of pie charts, each of the plot types may be used to plot up to four sets of data at the same time, each with a different plotting symbol (or no symbols at all if mathematical functions are to be plotted). For polygon plots, the continuity of the curves may be broken into blocks.

Labels and axes. Individual plots, axes within plots, points along axes, and individual curves may be labeled alphanumerically under user control. Axes may be scaled to the user's desires, and may have varying numbers of divisions (i.e., tick labels). Either axis or both may extend to negative values.

Data input and editing. PLTIT can handle large (13K, or over 2,000 points) data sets, and up to four data sets may be entered at the same time. Data may be entered from the keyboard or retrieved from disk. The program includes facilities for reviewing and editing the data. The data may be transformed before plotting without affecting the original data set so that various transformations may be attempted on the same data.

The author would like to thank J. Don Read and Keith Humphrey for their assistance in "user testing"' the program. The author's mailing address is: Department of Psychology, University of Lethbridge, Lethbridge, Alberta, Canada T1K 3M4.
Graph parameters and overlays. Individual plots may be located anywhere on the Apple computer's highresolution graphics screen, and may vary in both size and shape. Plots may be overlayed, one superimposed upon another (or many others), and multiple graphs, either of the same or different plot type and differing in size or shape, may be plotted in the same figure.

Printing graphs. PLTIT includes facilities for dumping completed plots (or any high-resolution picture) to a printer. Printing features include the options to invert (complement) the printed image, enhance (double-print) the image, and expand the printed image up to eight times in size along either or both the horizontal and vertical dimensions. The image may be cropped so that only particular areas will be dumped to the printer. Both serial and parallel printer interfaces are supported.

\section{System Requirements}

At a minimum, PLTIT requires a 48K RAM Apple II (or equivalent) computer, equipped with a single disk drive, with Applesoft BASIC in RAM (Apple II) or ROM (Apple II+, IIe, and IIc, or Franklin ACE, etc.). Optionally, multiple disk drives and a printer may be used. If the printer-driver within PLTIT is to be used to dump graphs to the printer, the printer must be an Apple DMP or an Apple ImageWriter (or equivalent-e.g., C. Itoh) connected to the computer via either a parallel or a serial interface that meets the minimum standards of an Apple parallel interface card or an Apple serial interface card. Alternatively, PLTIT-generated graphs may be saved to disk and dumped subsequently to a printer using the user's current picture-dumping routine.

\section{The PLTIT Package}

The complete PLTIT graphing package consists of the PLTIT program written in Applesoft BASIC, two machine-language files (one required to produce characters on the high-resolution screen, and the other to dump pictures to the printer), the source code of the machinelanguage picture-dumping routine, a stand-alone picturedumping program (PRNT.PIC) that incorporates all of the picture-printing features of PLTIT so that highresolution pictures may be dumped to a printer independently of PLTIT, and a user's manual. The package may be obtained without charge by sending an Applecompatible floppy diskette and a large self-addressed, stamped envelope to: John R. Vokey, Department of Psychology, University of Lethbridge, Lethbridge, Alberta, Canada T1K 3M4.

(Manuscript received for publication October 24, 1984.) 